\title{
2-D breathers and applications
}

\author{
J. L. Marín \\ J. C. Eilbeck \\ F. M. Russell
}

\begin{abstract}
In this chapter we show how a new type of nonlinear lattice excitation is helping to understand the long-standing puzzle of unexplained dark lines in crystals of muscovite mica. In fact, it was the conjecture that some kind of quasi-one-dimensional lattice soliton was responsible for those lines which led to the discovery of this new family of lattice excitations: mobile localized breathers of longitudinal type.

We explore several properties of these moving breathers, both by numerical methods and by experimenting with analogue models. The results suggest a much broader application than just the mica problem.
\end{abstract}

\section{Introduction}

There has been a long standing problem of understanding track formation in some mica minerals. Works by Russell and co-workers [25] had identified mysterious dark lines in natural crystals of muscovite mica, such as shown in Fig. 1. These are formed as a result of a local phase transition involving the precipitation of meta-stable dilutions of Fe and other impurity atoms. Track formation or recording processes of this type are normally thought to be triggered by energetic, charged particles (cosmic rays, particles from radioactive decay, etc.). However, most dark lines in mica cannot be explained in terms of charged particle tracks, although they closely resemble them. This prompted the conjecture of lattice solitons( "quodons") as the mechanisms forming the lines [20, 21].

Recent studies give strong support to this conjecture. In [14] we showed for the first time how localized excitations of longitudinal type can move in a 2-D lattice. These lattice modes appear to be the mobile version of intrinsic localized modes [24], more commonly known as discrete breathers. They are highly anharmonic and localized on just a few sites, and they propagate along lattice directions at sub-sonic speeds with a very small energy degradation. They are robust with respect to changes in the model, i.e., they appear in a wide range of lattices with different potentials. On the other hand they are not exact solitons, since they seem to interact nonelastically with other breathers. However, the loss of energy on collision frequently is small, so these pulses are reasonably robust.

We present here further studies of these moving breathers in systems with 


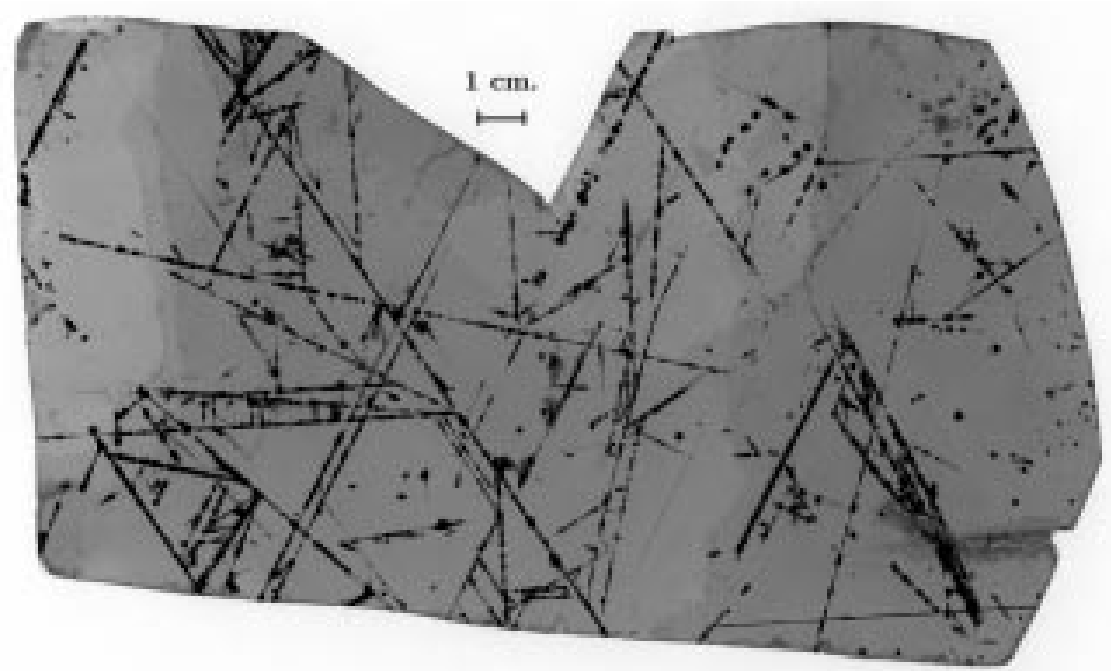

FIGURE 1. Photograph of a sheet of muscovite showing a wealth of tracks, most of them aligned along crystal directions.

realistic potentials, obtaining a more accurate model for the problem of tracks in mica. It is now recognized that the phenomenon is highly generic, and we point to two possible applications in the areas of condensed matter.

\section{Deciphering the lines in mica}

Natural crystals of muscovite mica often are doped with iron and other trace elements such as $\mathrm{Ca}, \mathrm{Mn}, \mathrm{Mg}$, and $\mathrm{Ti}$. In a similar way to carbon in metallic iron, the iron (Fe) in mica is held in solid solution at the high temperature (about 800K) needed for crystal formation. Such crystals are formed in solidifying magmas deep underground. As the crystals slowly cool, the solubility of Fe in mica decreases and, depending on the initial concentration, it may reach saturation. If this occurs then further slight cooling results in a super-saturated state in which the crystal structure is metastable. Given the opportunity via some type of localized lattice perturbation, the structure will revert to the lower-energy stable saturated state, precipitating the excess $\mathrm{Fe}$ as an oxide. With further slow cooling this solidstate phase transition continues until much of the Fe is eliminated from the mica lattice. During this later stage the precipitating Fe oxide accretes at the initial precipitation sites leading to massive growth or "decoration" of those sites. The rate of this decoration process is limited by the slow rate of release of $\mathrm{Fe}$ as the crystals cool slowly within the surrounding rock. The $\mathrm{Fe}$ oxide precipitates in the form of magnetite, $\mathrm{Fe}_{3} \mathrm{O}_{4}$, a moderate electrical 
conductor that absorbs light. This is why it appears as black spots, plates or ribbons in the otherwise clear mica crystal. Although the initial precipitation site is on the atomic scale, the decoration process can magnify them sufficiently as to make such ribbons visible to the unaided eye.

Although muscovite mica is a common mineral, it can form only under conditions of high temperature and pressure such as exist in molten pegmatite $5 \mathrm{Km}$ or more underground. With mountain building it erodes slowly to the surface and can be mined. Large nearly perfect crystals grow very slowly and it is not practical nor feasible to grow them in the laboratory for study of the initial triggering of the precipitation process. Hence, studies can be made only on samples of natural crystals in which the metastable conditions cannot be controlled. So the study of decorated defects in doped mica is a deductive science, like astronomy or cosmology. Nevertheless, progress in understanding the defects has been made by studying a wide variety of mica samples.

In the first studies of these tracks in mica, one of the authors (FMR, [17]) identified long decorated ribbons in the (001) planes, but in random directions. Many measurements of these tracks led to the conclusion that they had been created by energetic charged particles, such as muons created by cosmic rays, electron-positron showers created from these muons, and from positron emission coming from the decay of isotope ${ }^{40} \mathrm{~K}$ nuclei $[19,18]$. It was shown that the tracks from the ${ }^{40} \mathrm{~K}$ source were consistent with paths expected of charged particles following Rutherford scattering. The alternative explanation in terms of some type of crystal defect was highly improbable, given that the geometry of such defects would have to mimic the behavior of the charged particles paths.

But these were not the most interesting type of tracks observed in mica. In fact, most of the decorated lines could not be attributed either to the tracks of charged particles or to any other known type of crystal defect. These unexplained lines lie along crystal directions of low Miller indexes in the (001)-plane, although they show several features that resemble the tracks of charged particles. They can be scattered to branch off and create secondary and even tertiary tracks, and show decoration similar to that on charged particle tracks. A study of possible correlations between these unexplained lines and the tracks of charged particles showed that they did not occur in conjunction with positron tracks from ${ }^{40} \mathrm{~K}$ nuclei, but could occur in association with tracks of high-energy shower particles and also energetic muons. This suggested a dependence on the momentum of the charged particle, and led to the idea that they might result from atomic scattering events in the mica involving significant motion of atomic nuclei [23]. It was conjectured that energetic particles, if they possessed sufficient momentum, could create a new type of lattice excitation when scattered by one or more atoms of the lattice. Then this excitation would propagate through the crystal as a highly localized, particle-like entity. Atomic motions within this localized entity would influence the weak energy barrier that inhibited 
impurity precipitation, thereby triggering the recording process. The observations in mica showed that this entity had to possess a unique feature, namely, a remarkable localization or stability against lateral spreading as it propagated over great distances, even exceeding $500 \mathrm{~mm}$ in length in rare large crystals (see Fig. 1).

It was already known from the charged particle tracks that the track recording process responds only to particles or disturbances moving in the Potassium mono-atomic planes in mica. From these facts and a study of the mica crystal structure, it was concluded that the new kind of lattice excitation is associated with quasi-one-dimensional behavior in the $\mathrm{K}$ (001)-plane. To reflect this behavior the new entity or object was called a "quodon".

\section{Numerical and analogue studies}

To investigate the properties of these hypotherical qodons and their behavior in the mica, lattice studies were made, using both numerical (molecular dynamics) techniques and analogue models of coupled pendulums. Our aim is to model the nonlinear behavior of lattice motions of the $\mathrm{K}$ atoms, therefore it is important to understand the structure and the nature of the bonds involved.

In Fig. 2 we can see the crystal structure of muscovite. This mineral (chemical formula $\mathrm{KAl}_{2}\left(\mathrm{AlSi}_{3}\right) \mathrm{O}_{10}(\mathrm{OH})_{2}$ ) belongs to the family of micas, which in turn are a particular type of layer silicates. These are more commonly known as clays and clay-related minerals. The basic units forming these silicates are sheets of tetrahedra and octahedra (O atoms being the vertex and $\mathrm{Si}$ atoms being the centers of these). Fortunately, muscovite is not a very complicated silicate. It is a 2:1 silicate, with dioctahedral occupancy and with interlayer cation Potasium. This means that the layers are composed of one sheet of octahedra sandwitched between two sheets of tetrahedra, and the octahedral sheet has only two out of three octahedra occupied with a cation $\left(\mathrm{Si}^{+4}\right.$ or $\mathrm{Al}^{+3}$ in this case). The layer is negatively charged as a whole, due to the substitution of all $\mathrm{Si}$ atoms in the tetrahedral sheet by Al. Then the layers stack and bond through electrostatic forces, thanks to the presence of an interlayer cation sheet, composed of $\mathrm{K}^{+}$ ions. The crystal structure of muscovite has been studied experimentally in detail [16], and recently it has been modeled accurately with molecular mechanical methods [4].

We can see how the silicate layer forms a fairly rigid structure, with bonds which are predominantly covalent. The $\mathrm{K}$ atoms, though held by Coulomb forces in the direction perpendicular to the sheet, have easier motion on the sheet plane (the (001)-plane), only restrained by short-range forces. Therefore, a first approximation model consists of a 2D lattice of $\mathrm{K}$ atoms 

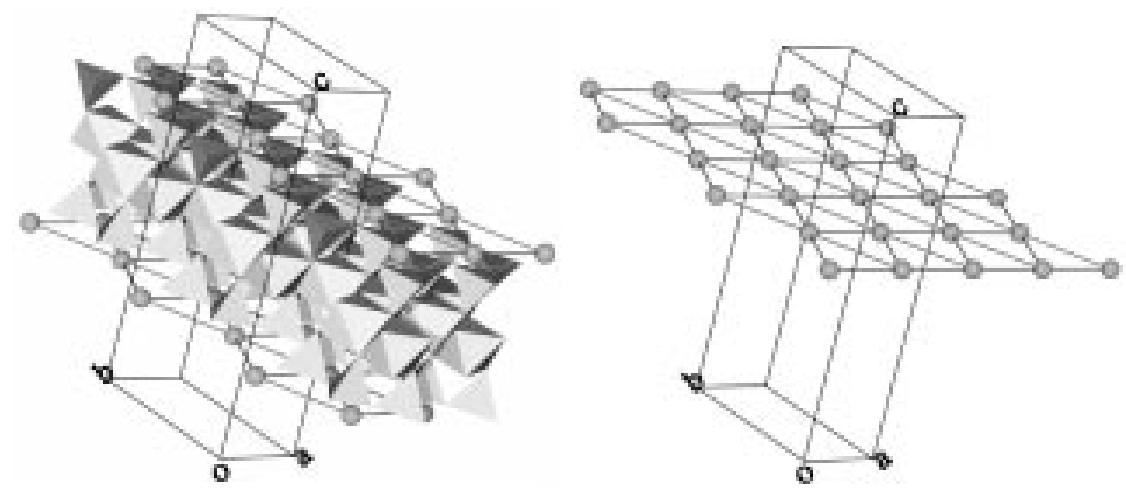

FIGURE 2. The structure of muscovite, emphasizing the potassium sheet.

subject to the action of an external "on-site" potential, constructed as to mimic the presence of a totally rigid silicate layer above and below.

The molecular dynamic techniques available at the time could not easily be used to study the behavior of the $\mathrm{K}$ lattice following a simulated impact on an atom by a projectile. To explore this problem mechanical analogues were made (by FMR) to simulate a one-dimensional chain of atoms subject to both inter-particle and on-site forces. In particular, the non-linearity of the forces in the analogues was made similar to those found from the molecular-mechanics studies. The analogue "atoms" were made from small permanent magnets that simulated the inter-particle forces. These magnets were suspended as rigid pendulums from fixed points and so used gravity to simulate the on-site forces. The strength of the inter-particle force could be varied by changing the spacing of the magnets along the chain. The strength of the on-site force could be changed by tilting the analogue away from a vertical position.

It was found that an impact on a particle in the chain quickly evolved into a traveling disturbance that retained its envelope shape. Within this envelope the particles executed anti-phase or optical-mode type oscillations, with a phase velocity that exceeded the group velocity of the envelope. The envelope was reflected if one particle in the chain was held fixed. Small perturbations of the chain, such as increasing the mass of one particle or the spacing between one pair, had little effect on the envelope. However, a large perturbation caused the envelope to separate into two of lower energy, one being reflected. By using a chain curved into a circle it was possible to follow such envelopes over many circuits round the circle. In summary, it was found that these localized excitations were remarkably robust and closely simulated the properties expected of the postulated quodon. The results were confirmed and expanded in detail by a numerical model of the analogue model itself [22]. Everything indicated that these solutions were moving breathers, which have attracted a great deal of research among 
the condensed matter and dynamical systems community (for a review, see $[9,1])$.

These one-dimensional models, however, could not account for the twodimensional nature of the problem. For instance, it was already known that Toda-like solitons in a two-dimensional sheet spread rapidly sideways, failing to propagate more than about a hundred atomic spacings [15]. As for discrete breathers, they had been found to be mobile in many 1D models [10, 5], but not in higher-dimensional cases [2] (the solutions in Ref. [26] seem too extended to qualify as discrete breathers).

We then set out to study numerically a full-blown $2 \mathrm{D}$ model, in which the $\mathrm{K}$ ions could move in the plane. The silicate layer is a complicated structure of tetrahedra and octahedra, and both surfaces of this layer are formed by the basal oxygens of the tetrahedra. These triangular bases are connected with each other at the vertices, and form an hexagonal honeycomb structure. The interlayer, the $\mathrm{K}$ sheet, is therefore sandwiched between mirror images of this structure, the atoms occupying the dimples left at the center of such honeycomb cells. The result is that the $\mathrm{K}$ sheet forms a perfect triangular lattice, with six-fold symmetry (see again Fig. 2). The on-site potential created by the silicate layer preserves this symmetry: the projections of the basal oxygens on the K-plane fall right at the midpoints between $\mathrm{K}-\mathrm{K}$ bonds, and the $\mathrm{Al}$ ions in the center of these tetrahedra project right on the center of the triangles formed by $\mathrm{K}$ ions ${ }^{1}$. In real mica crystals, there are always distortions to this ideal structure, like tetrahedral rotation. However, they normally preserve the six-fold symmetry for the environment of the $\mathrm{K}$ ions [4].

We studied several molecular-dynamics models of hexagonal 2D systems with different hexagonal symmetry, and eventually found solutions which perfectly fit with the quodon conjecture. More specifically, quasione-dimensional behavior is taken to mean that the excitation remains localized and moves along a favoured direction in the crystal, and that this phenomenon is assumed to be due to a particular type of crystal symmetry. This symmetry is such that if one of the atoms in the chain is disturbed from its equilibrium position along this favoured direction, then the restoring force is purely in the reverse direction to the displacement, i.e. there are no shear forces present [21]. The numerical 2D systems we studied have this property. The corresponding solitary waves that we found are moving discrete breathers of longitudinal type, and we describe in greater detail their properties in the next section.

\footnotetext{
${ }^{1}$ Although the $\mathrm{K}$ atoms form a triangular lattice, we have used sometimes the term "hexgonal" instead. We hope the reader does not mistake it for a honeycomb structure, which is not the case.
} 


\section{Longitudinal moving breathers in 2D lattices}

The numerical technique used in our models is purely classical molecular dynamics, although it is important to note that only first nearest neighbors are taken into account so far. The potentials used are those coming from molecular-mechanics studies, and although they are empirical, they are thought to be highly accurate for most modelling purposes.

However, for reasons to be shown shortly, it is not so important to get the detailed structure of the on-site potential to great accuracy, or even perfectly fitted interatomic potentials. We have settled for now with a model in which the $\mathrm{K}$ atoms interact through Lennard-Jones potentials,

$$
W_{L J}(r)=\frac{a^{2}}{72}\left(\left(\frac{a}{r}\right)^{12}-2\left(\frac{a}{r}\right)^{6}\right) ; \quad r \equiv|\vec{d}|
$$

where $\vec{d}=\vec{u}-\vec{u}^{\prime}-\vec{c}$ is the actual separation vector between the $(i, j)$ and the $\left(i^{\prime}, j^{\prime}\right)$-th nearest neighbor sites. We used as coordinates the relative displacements from the lattice equilibrium positions, $\vec{u}$, and $\vec{c}$ are static vectors connecting nearest-neighbor equilibrium sites. The particular scaling of this potential, as well as that of all others, was chosen so as to have a frequency for low-amplitude oscillations of $\omega=1$. The on-site potential is produced for each $\mathrm{K}$ atom by placing fixed Lennard-Jones atoms at positions above the plane, at the sites occupied by basal oxygens. For each $\mathrm{K}$ atom we took into account 12 oxygens: the 6 nearest neighbor ones (whose projections lie at midpoints of the $\mathrm{K}-\mathrm{K}$ bonds) and 6 next-nearest neighbor ones (with projections at midpoints of bonds between neighboring $\mathrm{K}$ atoms). Note that these fixed oxygens are really off the plane, so that the distances to the $\mathrm{K}$ atoms are computed in full 3D Euclidean space (the z-coordinate being constant). This is in contrast with our initial model in [14], where we placed 6 fixed atoms in the plane, interacting through a Morse potential.

Using the intuition gained about discrete breathers in 1D models, it was judged that it was not the particular shape of the nonlinear potentials used which was of main importance. Rather, it was the relative strength between the inter-atomic and on-site potentials which was crucial for the existence of these solutions. In the simpler case of stationary breathers, it had been established rigorously how discrete breathers emerge from the limit of negligible inter-atomic potential (the so-called anti-continuum limit, see [11]). The case of moving breathers is much less understood, but it has been shown how one can tune the relative strengths of on-site and inter-particle potentials and eventually find regions of easy breather mobility [5]. These regions appear to happen near bifurcations which connect the site-centered and bond-centered stationary breathers (see Ref. [12]), and the subject is being investigated in greater detail [6]. It is difficult to estimate how wide these regions are, other than by means of numerical simulation. 
With these heuristics we found moving longitudinal breathers in our simulations. Just like in 1D models, it was observed that a too strong on-site potential hinders mobility, and favours the pinning of the excitations into stationary breathers (intrinsic localized modes). A too weak on-site potential usually destroys the breather by broadening and radiation into the background, due to resonances with the phonon band [12]. In between these extremes, there exists an ample region where it is easy to obtain moving breathers, with a more or less wide range of energies and velocities. The striking novelty here is that these breathers have a strong transversal focusing effect: the excitation is a sharply localized longitudinal wave, comprised of about 4-8 particles in the longitudinal direction, but practically only one particle in the transverse direction. Note that the focusing effect is clearly not an artifact of weak couplings: if one tries to create a moving breather under a certain energy threshold, the excitation dies out radiating in all crystal directions in a linear-like fashion. It all indicates that these breathers are the realization of the quodon conjecture.

Fig. 3 shows the profile of the longitudinal displacements of the $\mathrm{K}$ atoms along the breather path. These solutions travel very long distances along lattice directions, with negligible loss of energy by radiation. As in the analogue studies, the solutions are characterized by nearly out-of-phase motion of the atoms involved, with a frequency which seems to be independent of the envelope wave velocity. The internal vibration frequency is high (above the linear phonon band, due to the effective hardening nonlinearity), while the breather motion is slow, typically $20-60 \%$ of the sound velocity in the lattice. This indicates that they are truly moving breathers, with two dynamical degrees of freedom. Other types of lattice solitons, where carrier wave and soliton velocity go in unison, can be calculated with accurate procedures, such as continuation methods [8]. In some special cases, such as the discrete nonlinear Schrödinger equation, the same methods can be applied to calculate breathers [7]. But in general, moving breathers are much harder to obtain in such detail, and although some approaches have proved useful for very slow ones [5], we do not have accurate methods for most of the range of possible solutions observed in simulations.

As we mentioned above, our studies are based on heuristics and numerical simulation. However, the way moving breathers are produced in the simulation is very useful for the problem of mica. Instead of trying to isolate and "purify" a moving breather, it was interesting to know how they could be formed. It turned out that it is very easy to produce them, simply by giving some atoms certain amounts of energy. In practice, it is more controllable to give the particles an initial velocity impulse, as if an atomic collision took place. It was surprising to observe that giving an impulse to just one atom is enough to create the moving breather. There is initially a burst of radiation away from the disturbance, but once the breather emerges it sheds very little radiation and may travel tens of thousands of sites without appreciable degradation. Furthermore, there is robustness against deflec- 


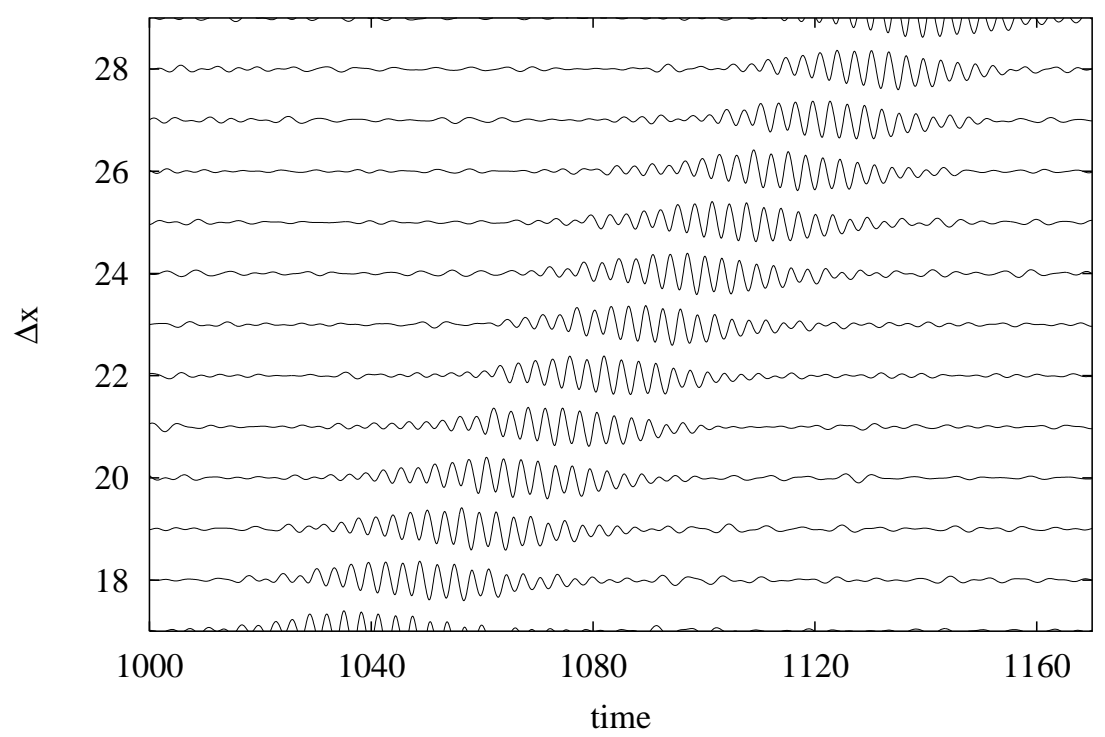

FIGURE 3. Longitudinal displacements vs. time for the atoms on the breather track. They have been scaled by a factor of five to make them more visible; actual displacements reach about $15-20 \%$ of the interatomic distance. 
tions of this initial momentum: we found that deviations as great as $15^{\circ}$ from the crystal axis would still produce a breather.

In summary, these longitudinal breathers are a very robust phenomenon appearing in 2D lattices, in the sense that they do not need a special shape for the potentials, or a very constrained range of parameters. We have found them in several types of Lennard-Jones, Morse and other lattices, within ample windows of parameter ranges. A common feature is the need for an on-site potential, although some recent results suggest that it is possible to reach the limit of null on-site terms in some particular models. After all, the exact extent of the parameter ranges in which these breathers exist will vary from one model to another, and the intrinsic nonlinear nature of the problem makes it difficult to predict it a priori. For the problem of mica, we are now trying to introduce the most realistic potentials we can find [4] and check whether the combination of on-site and inter-atomic forces for the $\mathrm{K}$ atoms allow for moving breathers. Our initial studies including only short range forces suggest so [13]. It is now needed to study the effect of long-range Coulombic forces on these moving solutions, which is not well known even for 1D models.

\section{Breather Collisions}

We have experimented with a number of different configurations to study breather-breather collisions. Two breathers can collide head-to-head inline, or head-to-head but on parallel tracks, or at angles of $60^{\circ}$ or $120^{\circ}$. In the latter case the collision can also be "head-on" or staggered (i.e. one breather just leaving a particular site as the other is arriving). The results will also depend on the relative phases of the underlying carrier waves and on the energies of each breather. The number of possible permutations are clearly large and will be described in more details elsewhere. We give here just one example, an in-line head-to-head collision beween two breathers of equal amplitude, as shown in Fig. 4, a-d.

In this case we see that the two breathers are reflected almost elastically with very little radiation, either transverse or longitudinal. However this result is very dependent on the relative phases of the breathers, with a slightly different starting position (1 lattice spacing futher apart), the two breathers coalesce into a stationary "super-breather", two breathers bound together but with a time-periodic small separation which decreases slowly with time.

These results just confirm that discrete breathers are not exact solitons, and therefore they might not always have the expected robustness with respect to collisions. On the other hand, they have a different kind of robustness, that which is conferred by their high genericity. Exact solitons appear in integrable models, but discrete breathers appear generically in 

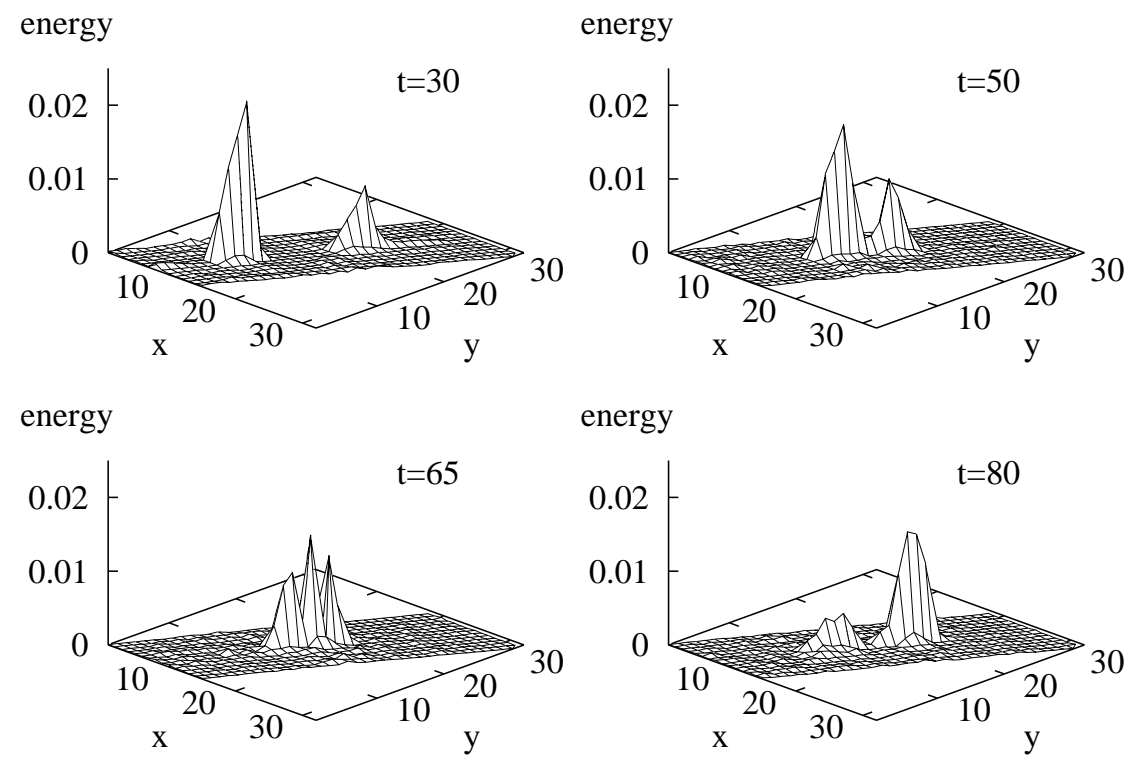

FIGURE 4. Head-on collision of two breathers with different energies and frequencies, in an hexagonal lattice. In this case they emerge out of the collision almost unaffected. 
an extremely large class of lattices.

\section{Conclusions and further applications}

It does not pass unnoticed that such a generic phenomenon as these lattice breathers should have applications to many other fields, and not just mica structures. Here we sample some of our speculations with respect to the ubiquity of these new solutions.

\subsection{Application to sputtering}

These successes in simulating the predicted quodons and their behavior prompted application of the quodon concept, in particular, to sputtering at a crystal surface and to other crystals with a layered structure. Sputtering, the ejection of atoms from a crystal surface that is bombarded by energetic particles, has a long history and is of industrial importance. Pioneering work was done by Wehner in 1956 [27], who showed that sputtering was intimately related to the speed of sound in a material. He found also that sputtered atoms were ejected in atomic-chain directions and that particles bombarding a surface at normal incidence gave a higher energy threshold for sputtering than at oblique incidence. We believe that the new found moving breathers could bring many new insights into this old subject, which has never been given detailed atomistic treatment. Initial experiments carried out with our analogue models are finding agreement with the phenomenological theories of Wehner, and indicate the importance of understanding the nonlinear dynamics of lattices.

\subsection{Application to layered HTSC materials}

The discovery of high temperature superconductivity (HTSC) in layered cuprates raised the question of the possibility of quasi-one-dimensional behavior in those layers. A similar molecular dynamic study to that of the mica system was made of several known HTSC materials containing cuprate layers to find out if any of the many different layers might be capable of allowing quasi-one-dimensional behavior. The materials examined were $\mathrm{YBa}_{2} \mathrm{Cu}_{3} \mathrm{O}_{7}, \mathrm{Y}_{2} \mathrm{Ba}_{4} \mathrm{Cu}_{6} \mathrm{O}_{13}$, and $\mathrm{La}_{2} \mathrm{CuO}_{4}$. It was found that in all three materials movement of $\mathrm{Cu}$ and $\mathrm{O}$ atoms in chain directions in the non-puckered (001)-planes was one-dimensional like in that no shear forces were created in the surrounding lattice [21]. Some Y atoms also showed quasi-one-dimensional behavior. These findings suggest that qodons might be created and propagate in such materials.

The dominant mechanism of charge-pair coupling in HTSC is still not known. However, it has been shown recently that in the HTSC materials 
$\mathrm{Bi}_{2} \mathrm{Sr}_{2} \mathrm{Ca}_{m-1} \mathrm{Cu}_{m} \mathrm{O}_{y},(m=1$ and 2$)$ the $\mathrm{T}_{\mathrm{c}}$ is independent of the separation distance between the cuprate layers [3] and is the same as that for a single layer. This strongly suggests that the pairing mechanism operates in a 2D layer. From study of the published structures of these two materials it seems both may show quasi-one-dimensional properties in certain layers. This needs to be examined in detail.

In order to investigate the possibilities of breathers mediating the coupling effects on charges in HTSC in tetragonal lattices, we have carried out a similar study to the present paper but using square 2D lattices instead of hexagonal lattices. We find localized breather solutions for a wide range of model parameters and initial disturbances. These results will be reported elsewhere.

Acknowledgments: JLM acknowledges a postdoctoral Marie Curie TMR fellowship from the EC (grant no. ERBFMBICT972761). We would also like to thank Paz Vaqueiro for providing us with accurate data for the crystal structures.

\section{REFERENCES}

[1] S. Aubry. Breathers in nonlinear lattices: existence, linear stability and quantization. Physica D, 103:201-250, 1997.

[2] V. M. Burlakov, S. A. Kisilev, and V. N. Pyrkov. Computer simulation of intrinsic localized modes in one-dimensional and two-dimensional anharmonic lattices. Phys. Rev. B, 42:4921, 1990.

[3] Jin-Ho Choy, Soon-Jae Kwon, and Gyeong-Su Park. High $T_{c}$ superconductors in the two-dimensional limit. Science, 280:1589-1592, 1998.

[4] D. R. Collins. Computer simulations and netron scattering studies of layer silicates minerals. PhD thesis, University of Keele, 1990.

[5] T. Cretegny and S. Aubry. Mobility and reactivity of discrete breathers. Physica D, 119:34-46, 1998.

[6] T. Cretegny, S. Aubry, and J. L. Marín. Exchange of stability and mobility of discrete breathers. in preparation.

[7] D. B. Duncan, J. C. Eilbeck, H. Feddersen, and J. A. D. Wattis. Solitons on lattices. Physica D, 68:1-11, 1993.

[8] J. C. Eilbeck and R. Flesch. Calculation of families of solitary waves on discrete lattices. Phys. Lett. A, 149(4):200-202, 1990.

[9] S. Flach and C. R. Willis. Discrete breathers. Physics Reports, 295:181-264, 1998. 
[10] K. Hori and S. Takeno. Low-frequency and high-frequency moving anharmonic localized modes in a one-dimensional lattice with quartic anharmonicity. J. Phys. Soc. Jpn., 61:4263, 1992.

[11] R. S. MacKay and S. Aubry. Proof of existence of breathers for timereversible or Hamiltonian networks of weakly coupled oscillators. Nonlinearity, 7:1623-1643, 1994.

[12] J. L. Marín, S. Aubry, and L. M. Floría. Intrinsic localized modes: discrete breathers. existence and linear stability. Physica D, 113:283$292,1998$.

[13] J. L. Marín, J. C. Eilbeck, and F. M. Russell. Localized moving breathers in 2-d lattices. in preparation.

[14] J. L. Marín, J. C. Eilbeck, and F. M. Russell. Localized moving breathers in a 2-d hexagonal lattice. Phys. Lett. A, 1998. to appear.

[15] Yu V. Martynenko and P. G. Moscovkin. Solitons in radiation physics of crystals. Radiation Effects and Defects in Solids, 117:321-328, 1991.

[16] E. W. Radoslovich. The structure of muscovite, $\mathrm{KAl}_{2}\left(\mathrm{Si}_{3} \mathrm{Al}\right) \mathrm{O}_{10}(\mathrm{OH})_{2}$. Acta Cryst., 13:919-930, 1960.

[17] F. M. Russell. The observation in mica of charged particles from neutrino interactions. Phys. Lett. B, 25:298-300, 1967.

[18] F. M. Russell. Identification and selection criteria for charged lepton tracks in mica. Nucl. Tracks Radiat. Meas., 15:41-44, 1988.

[19] F. M. Russell. Positive charge transport in layered crystalline solids. Phys. Lett. A, 130:489-491, 1988.

[20] F. M. Russell and D. R. Collins. Lattice-solitons in radiation damage. Nuclear instruments $\&$ methods in physics research B, 105:1-4, 1995.

[21] F. M. Russell and D. R. Collins. Anharmonic excitations in high $T_{c}$ materials. Phys. Lett. A, 216:197-202, 1996.

[22] F. M. Russell, Y. Zolotaryuk, and J. C. Eilbeck. Moving breathers in a chain of magnetic pendulums. Phys. Rev. B, 55:6304-6308, 1997.

[23] D. Schlosser, K. Kroneberger, M. Schosnig, F. M. Russell, and K. O. Groeneveld. Search for solitons in solids. Radiat. Meas., 23:209-213, 1994.

[24] A. J. Sievers and S. Takeno. Intrinsic localized modes in anharmonic crystals. Phys. Rev. Lett., 61:631-633, 1988. 
[25] J. W. Steeds, F. M. Russell, and W. J. Vine. Formation of epidote fossil positron tracks in mica. Optik, 92:149-154, 1993.

[26] J. M. Tamga, M. Remoissenet, and J. Pouget. Breathing solitary waves in a sine-Gordon two-dimensional lattice. Phys. Rev. Lett., 75:357361, 1995 .

[27] G. K. Wehner. Controlled sputtering of metals by low-energy Hg ions. Phys. Rev., 102:690-704, 1956. 